\title{
de novo MAPT mutation G335A causes severe brain atrophy, 3R and 4R PHF-tau pathology and early onset frontotemporal dementia
}

\author{
Kunie Ando ${ }^{1 *}$, Lorenzo Ferlini ${ }^{2}$, Valérie Suain ${ }^{1}$, Zehra Yilmaz ${ }^{1}$, Salwa Mansour ${ }^{1}$, Isabelle Le Ber ${ }^{3}$, Cécile Bouchard ${ }^{4}$, \\ Karelle Leroy ${ }^{1}$, Alexandra Durr ${ }^{3}$, Fabienne Clot $^{5}$, Marie Sarazin ${ }^{6}$, Jean-Christophe Bier ${ }^{2}$ and Jean-Pierre Brion ${ }^{1 *}$ (D)
}

Keywords: Neurofibrillary tangles, Tau pathology, FTLD-tau, MAPT

\section{Main text}

Tauopathies are neurodegenerative disorders characterized by hyperphosphorylated microtubule-associated protein tau (MAPT) forming protein aggregates. Over sixty dominantly inherited mutations in MAPT have been reported. These mutations cause frontotemporal lobar degeneration with tau-immunoreactive inclusions (FTLD-tau) whose symptom onset and survival are highly heterogeneous [4]. We describe here the genetic, clinical, neuropathological and biochemical characterization of a case of de novo MAPTG335A mutation in a male patient.

Symptoms of poor organisation skills, attention and concentration difficulties appeared when the proband was 14 years old. A few years later, he developed anxiety, panic attacks and time obsession. Firstly, he was considered to be suffering from a schizophrenic disorder. Since behavioral and phasic disturbances progressivly appeared, he was referred to a department of neurology at the age of 24. By this time, MRI showed a frontotemporal atrophy and SPECT demonstrated an hypofixation in the same regions. The clinical evolution was marked by a progressive decline in cognitive functions, particularly in speech output, semantic memory and in

\footnotetext{
* Correspondence: Kunie.Ando@ulb.ac.be; jpbrion@ulb.ac.be 'Laboratory of Histology, Neuroanatomy and Neuropathology, Faculty of Medicine, Université Libre de Bruxelles, ULB Neuroscience Institute, 808 route de Lennik, Bldg GE, B-1070 Brussels, Belgium

Full list of author information is available at the end of the article
}

executive functions, an hyperorality, an emotional blunting and a buccofacial apraxia with swallowing disturbance necessiting an institutionalisation in an appropiate facility when he was 25 . He never developed major extrapyramidal features, except a dystonia of upper left arm and a slight upper arms rigidity probably of iatrogenic origin (i.e. neuroleptic drugs).

Sequencing of $M A P T$ gene revealed a novel heterozygous mutation G335A due to $\mathrm{G} \rightarrow \mathrm{C}$ transition at the second base position in codon 335 in exon 12 (NM 005910.5: c.1004G > C p.Gly335Ala). The mutation was not found in his parents, thus strongly arguing for a de novo mutation. The proband died at 34, 20 years after the onset of symptoms. The proband's brain was removed at autopsy, collected and stored in the brain bank of the LHNN (BB190052). One brain hemisphere was fixed in $10 \%$ buffered formalin. The other hemisphere was cut into coronal sections and kept at $-80^{\circ} \mathrm{C}$. A small piece of frontal cortex was fixed in $4 \%$ glutaraldehyde for transmission electron microscopy.

The macroscopic examination showed a severe atrophy of the frontal and anterior temporal lobes (Fig. 1a) with a whole wet brain weight of $928 \mathrm{~g}$. A marked dilatation of the frontal horn of the lateral ventricule was present (Fig. 1b). Neuropathological analysis was done on paraffin-embedded sections. In all regions examined, there were neurodegenerative changes consistent with a tauopathy (Table 1). Possible neuropathological 


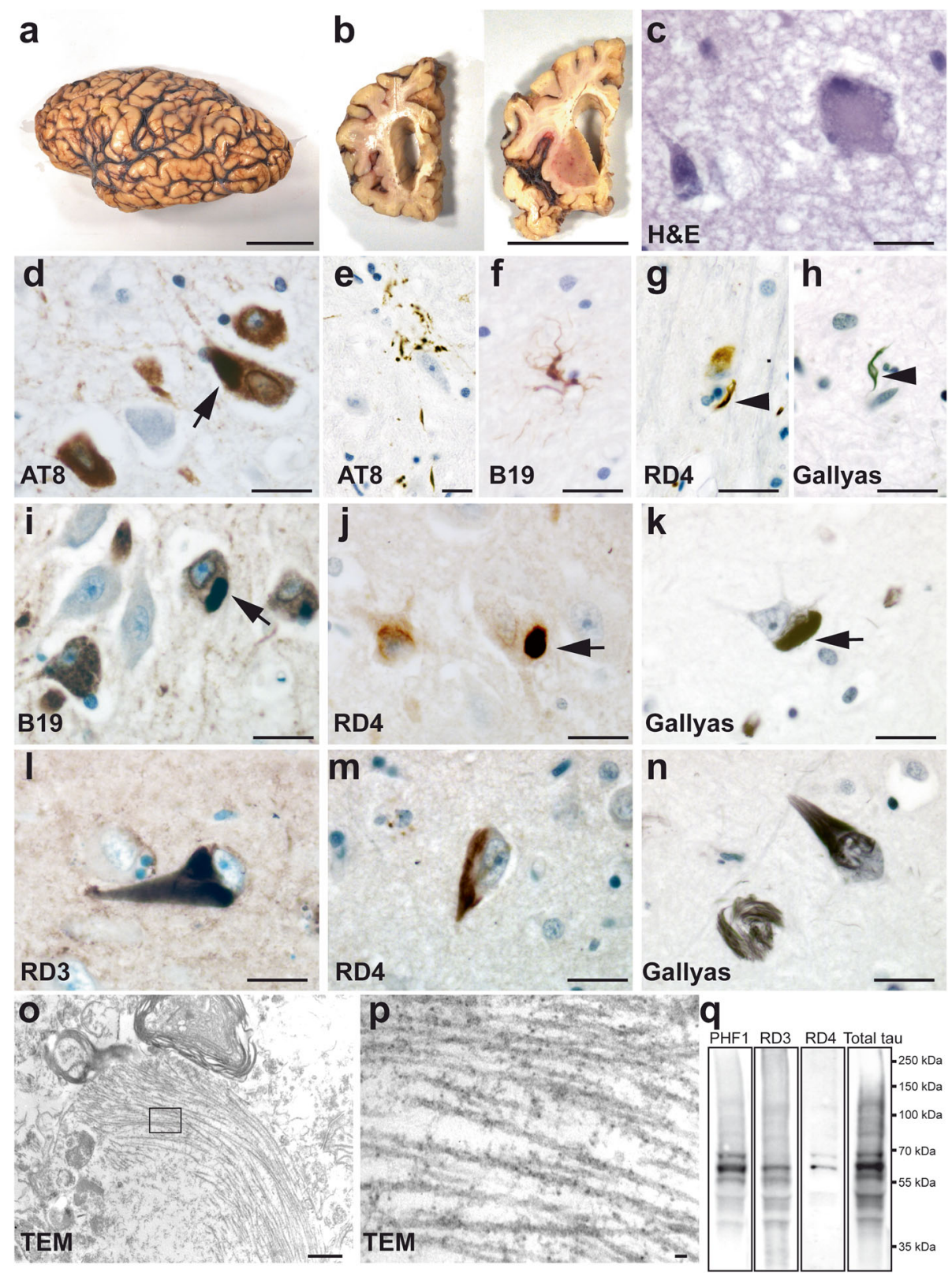

Fig. 1 de novo mutation of MAPT-G335A led to severe brain atrophy with glial and neuronal tau pathology constituted of 3R and 4R tau. a Macroscopic pathology of FTLD-tau with MAPT-G335A mutation. The fixed left cerebral hemisphere showed a severe circumscribed atrophy of frontal lobe and anterior temporal lobe (with sparing of posterior superior gyrus). Motor and sensory gyri and occipital lobe were relatively spared. $\mathbf{b}$ Coronal sections of fixed brain. The frontal horn of the lateral ventricle was markedly dilated. $\mathbf{c}$ H\&E reveals a ballooned neuron (right) and microvacuolation. d-e Immunostaining with AT8 (Thermo Scientific) (pS202/T205 tau) revealed numerous NFT in the pyramidal layer of the hippocampal CA1-2 sector (d). Occasionally there were astrocytic plaque-like appearance of tau-positive deposits in the striatum, thalamus and temporal cortex (e). f B19 (total tau)-positive astrocyte, reminiscent of tufted astrocytes were observed in the thalamus, frontal cortex and temporal cortex. $\mathbf{g}$ Coiled bodies (arrowhead) were immunolabelled by RD4 (clone 1E1/A6, Millipore) (4R tau). $\mathbf{h}$ Coiled bodies (arrowhead) were Gallyas positive. i-k Pyramidal neurons of the hippocampal CA2-3 sector immunostained for anti-tau B19 (i), RD4 (4R tau) (j) and Gallyas staining (k). Arrows show intraneuronal 4R tau positive globular and elongated inclusions (d, i-k). I-n NFT were immunostained with RD3 (clone 8E16/C11, Millipore, I) (3R tau) and RD4 (4R tau) ( $\mathbf{m})$. $\mathbf{n}$ Gallyas staining in CA2 sector shows a classical NFT (right) and Gallyas-positive intraneuronal threads (left). o-p Transmission electron microscopy showing a cytoplasmic aggregate of filaments in the frontal cortex. At higher magnification (p) of the inset in o, both straight filaments and PHF were observed. o 12,000x; p 30,000x. q Sarkosyl insoluble tau from the frontal cortex of the proband (MAPT-G335A) immunoblotted with PHF1, RD3, RD4 and total tau (A0024, Dako) antibodies. Sarkosyl insoluble tau is constituted of hyperphosphorylated $3 R$ and $4 R$ tau. Scale bars, $5 \mathrm{~cm}$ for $\mathbf{a}-\mathbf{b}$; $20 \mu \mathrm{m}$ for $\mathbf{c}-\mathbf{n} ; 500 \mathrm{~nm}$ for $\mathbf{o}$ and $30 \mathrm{~nm}$ for $\mathbf{p}$ 
Table 1 Semi-quantitative assessment and regional distribution of tau pathology on brain sections immunolabelled with AT8. -: absent; +: occasional; ++: moderate number; +++: frequent number. NFT, neurofibrillary tangles

\begin{tabular}{|c|c|c|c|c|}
\hline \multirow[b]{2}{*}{ Brain areas } & \multicolumn{4}{|c|}{ Tau pathology } \\
\hline & NFT & Neuronal diffuse & Oligodendroglial & Astrocytic \\
\hline Hippocampal pyramidal layer & +++ & +++ & ++ & + \\
\hline Dentate gyrus & - & + & - & - \\
\hline Subiculum & ++ & ++ & + & + \\
\hline Transentorhinal cortex & + & + & + & + \\
\hline Temporal cortex & ++ & ++ & ++ & + \\
\hline Frontal cortex & ++ & + & ++ & + \\
\hline Cingulate cortex & + & + & + & + \\
\hline Visual cortex & - & - & - & - \\
\hline Striatum & ++ & + & +++ & + \\
\hline Globus pallidus & ++ & + & + & + \\
\hline Thalamus & ++ & + & + & + \\
\hline Substantia nigra & +++ & + & + & + \\
\hline Pontine nuclei & ++ & + & + & + \\
\hline Olivary Nuclei & - & + & - & - \\
\hline
\end{tabular}

comorbidity was excluded by immunohistochemistry for $\mathrm{A} ß, \alpha$-synuclein and TDP-43. Significant neuronal loss and microvacuolation were observed in the superior layers of frontal and temporal cortex, where some ballooned neurons were also observed (Fig. 1c). In the deep layers of frontal and temporal cortex, there were neurofibrillary tangles (NFT) with flame shaped or globular forms, pre-tangles, neuropil threads, grain-like neuropil threads that were immunolabelled for total tau (B19) [2] and phosphotau AT8. Some NFT were detected in the parietal cortex but not in occipital cortex. NFT were prominent in the hippocampal CA sector and in the subiculum (Fig. 1d). Some astrocytes contained granular or punctate tau-immunoreactive deposits reminiscent of astrocytic plaques and tufted astrocytes (Fig. 1e, f). Tauimunoreactive astrocytes were predominantly $4 \mathrm{R}$ tau positive (Supplementary figure 1a-c, online resource). There were numbers of tau-positive coiled-bodies and axons in the white matter and in the deep cortical layers. The coiled bodies were labelled by $4 \mathrm{R}$ tau antibody and by Gallyas staining (Fig. 1g-h). Neuronal loss and NFT were prominent in substantia nigra. In the brainstem, tau positive lesions were detected in the pontine nuclei and in the olivary nuclei of the medulla. Some pyramidal neurons in hippocampal CA contained globular and elongated tau inclusions positive for $4 \mathrm{R}$ tau and Gallyas (Fig. 1d, i-k). NFT were detected by anti-3R and $4 R$ tau antibodies (Fig. 1l, m). Some neurons had Gallyaspositive intraneuronal linear thread-like structures (Fig. 1n). GFAP positive gliosis was remarkable in the superior layers of the frontal cortex but less in the temporal cortex. A significant gliosis was observed in the sub- ependymal zone, especially at the level of the striatum and under the pia of the brainstem.

The ultrastructural aspect of the tau inclusions was analysed on ultrathin sections by transmission electron microscopy (Fig. 1o). Fibrillar inclusions were composed of both straight and paired helical filament (PHF) (Fig. 1p).

The sarkosyl fractionation method was used to enrich the insoluble tau from frozen grey matter of the frontal cortex [1] (Fig. 1q). By immunobloting, the sarkosyl insoluble tau extracted from the proband showed three major bands of 60,64 and $68 \mathrm{kDa}$ and was constituted of both $3 \mathrm{R}$ and $4 \mathrm{R}$ tau isoforms.

This is the first report of the G335A mutation in exon 12 of $M A P T$ that caused a strikingly early onset disease at 14 years old. Previously two MAPT mutations on the same amino acid have been reported to cause early onset frontotemporal dementia: the age of onset was 22 years old for $M A P T-G 335 \mathrm{~V}$ [7] and 25.4years old on average for MAPT-G335S [10]. Compared to other FTLD-tau-MAPT cases with an average age at onset of 49 years [4, 8], mutations at MAPT-G335 led to early onset and severe neuronal and glial tau pathology ultrastructurally composed of straight filaments and PHF in MAPT-G335A (this study) and in MAPT-G335S [10]. The MAPT-G335S mutation was associated to neuronal tau pathology (tau positive neurons, NFT, neuropil threads) and glial tau pathology (coiled bodies, tau positive astrocytes), similarly to what we observed here in the MAPT-G335A mutation. Neuronal inclusions in the present MAPT-G335A were labelled with both anti-3R tau and anti-4R tau antibodies, but 3R tau positive Pick bodies were not detected, in contrast 
with other MAPT mutations such as G272V [3] and L266V [5]. The immunobloting pattern of sarkosylinsoluble tau in MAPT-G335A is similar to Alzheimer's disease [9]; the presence of both $3 R$ and $4 R$ tau isoforms in the insoluble fraction was also observed in MAPTG389R [6] and MAPT-L266V [5]. This MAPT-G335A mutation changes the third among the four invariant PGGG motif in the microtubule-binding region to PGGA, and is predicted to affect the binding of tau to microtubules. Indeed, G335V and G335S mutations were reported to strongly affect this binding $[7,10]$, a molecular effect that might lead to increased level of free tau and the assembly of tau into filaments.

\section{Supplementary information}

Supplementary information accompanies this paper at https://doi.org/10. 1186/s40478-020-00977-8.

Additional file 1: Figure S1. Tau positive astrocytes reminiscent of tufted astrocytes were predominantly detected by anti-4R tau RD4 antibody in the temporal cortex of MAPT-G335A brain. 4R tau isoform (green) is expressed in an astrocyte immunolabelled by the astrocyte marker GFAP (red). The mouse monoclonal anti-4R tau RD4 antibody (clone 1E1/ A6, Millipore) and the rabbit polyclonal anti-GFAP antibody (Sigma G9269) were detected as previously described [1]

\section{Abbreviations}

MAPT: Microtubule-associated protein tau; MRI: Magnetic Resonance Imaging; TDP-43: TAR DNA-binding protein 43; NFT: Neurofibrillary tangles; SPECT: Single-photon emission computed tomography; CA: Cornu Ammonis; 3R tau: Three-repeat tau isoform; 4R tau: Four-repeat tau isoform; PHF: Paired helical filament

\section{Acknowledgements}

We thank Dr. Peter Davis (The Feinstein Institute for Medical Research, New York) for PHF1 antibody.

\section{Authors' contributions}

$K A, V S, Z H, S M, K L$, JPB performed neuropathological analyses, KA and JPB drafted the manuscript. $L F, I L B, C B, A D, F C, M S$, JCB contributed to acquisition and/or analysis of the clinical data. All authors have read and approved the final manuscript.

\section{Funding}

JPB was supported by grants from the Belgian Fonds de la Recherche Scientifique Médicale (T.0023.15, T0027.19), the Fund Aline (King Baudoin Foundation). JPB and $\mathrm{KL}$ were supported by the Belgian Alzheimer Research Foundation (SAO-FRA)

\section{Availability of data and materials}

Not applicable.

\section{Ethics approval and consent to participate}

Autopsy and gene analysis were carried out after written informed consent obtained from family members. The study using the post-mortem tissue was performed in compliance and following approval of the Ethical Committee of the Medical School of the Free University of Brussels.

\section{Consent for publication}

Family members have consented to publication.

\section{Competing interests}

The authors declare that they have no competing interests.

\section{Author details}

${ }^{1}$ Laboratory of Histology, Neuroanatomy and Neuropathology, Faculty of Medicine, Université Libre de Bruxelles, ULB Neuroscience Institute, 808 route de Lennik, Bldg GE, B-1070 Brussels, Belgium. ²Department of Neurology, Université Libre de Bruxelles, Hôpital Erasme, 808 route de Lennik, B-1070 Brussels, Belgium. ${ }^{3}$ Sorbonne Université, Institut du Cerveau (ICM) - Paris Brain Institute, AP-HP, INSERM, CNRS, University Hospital Pitié-Salpêtrière, Paris, France. ${ }^{4}$ Boulogne Billancourt, France. ${ }^{5}$ Université Paris-Saclay, CEA, CNRS, Inserm, BioMaps, Orsay, France. ${ }^{6}$ Unit of Neurology of memory and language, Groupe Hospitalier Universitaire Paris - Psychiatrie et Neurosciences, Hôpital Sainte Anne, Université de Paris, Paris, France and Université Paris-Saclay, CEA, CNRS, Inserm, BioMaps, 91401 Orsay, France.

Received: 11 May 2020 Accepted: 21 June 2020

Published online: 29 June 2020

\section{References}

1. Ando K, Brion JP, Stygelbout V, Suain V, Authelet M, Dedecker R et al (2013) Clathrin adaptor CALM/PICALM is associated with neurofibrillary tangles and is cleaved in Alzheimer's brains. Acta Neuropathol 125:861-878

2. Brion JP, Hanger DP, Bruce MT, Couck AM, Flament-Durand J, Anderton BH (1991) Tau in Alzheimer neurofibrillary tangles. N- and C-terminal regions are differentially associated with paired helical filaments and the location of a putative abnormal phosphorylation site. Biochem J 273(Pt 1):127-133

3. Bronner IF, ter Meulen BC, Azmani A, Severijnen LA, Willemsen R, Kamphorst W et al (2005) Hereditary Pick's disease with the G272V tau mutation shows predominant three-repeat tau pathology. Brain 128:2645-2653

4. Greaves CV, Rohrer JD (2019) An update on genetic frontotemporal dementia. J Neurol 266:2075-2086

5. Hogg M, Grujic ZM, Baker M, Demirci S, Guillozet AL, Sweet AP et al (2003) The L266V tau mutation is associated with frontotemporal dementia and pick-like 3R and 4R tauopathy. Acta Neuropathol 106:323-336

6. Murrell JR, Spillantini MG, Zolo P, Guazzelli M, Smith MJ, Hasegawa M et al (1999) Tau gene mutation G389R causes a tauopathy with abundant pick body-like inclusions and axonal deposits. J Neuropathol Exp Neurol 58: 1207-1226

7. Neumann M, Diekmann S, Bertsch U, Vanmassenhove B, Bogerts B, Kretzschmar HA (2005) Novel G335V mutation in the tau gene associated with early onset familial frontotemporal dementia. Neurogenetics 6:91-95

8. Reed LA, Wszolek ZK, Hutton M (2001) Phenotypic correlations in FTDP-17. Neurobiol Aging 22:89-107

9. Spillantini MG, Crowther RA, Goedert M (1996) Comparison of the neurofibrillary pathology in Alzheimer's disease and familial presenile dementia with tangles. Acta Neuropathol 92:42-48

10. Spina S, Murrell JR, Yoshida H, Ghetti B, Bermingham N, Sweeney B et al (2007) The novel tau mutation G335S: clinical, neuropathological and molecular characterization. Acta Neuropathol 113:461-470

\section{Publisher's Note}

Springer Nature remains neutral with regard to jurisdictional claims in published maps and institutional affiliations.

\section{Ready to submit your research? Choose BMC and benefit from}

- fast, convenient online submission

- thorough peer review by experienced researchers in your field

- rapid publication on acceptance

- support for research data, including large and complex data types

- gold Open Access which fosters wider collaboration and increased citations

- maximum visibility for your research: over $100 \mathrm{M}$ website views per year

At BMC, research is always in progress.

Learn more biomedcentral.com/submission 